\title{
Bevacizumab terminates homeobox B9-induced tumor proliferation by silencing microenvironmental communication
}

\author{
Yoshinori Hoshino ${ }^{1}$, Tetsu Hayashida ${ }^{1 *}$, Akira Hirata ${ }^{1}$, Hidena Takahashi ${ }^{1}$, Naokazu Chiba ${ }^{1}$, Mitsuyo Ohmura ${ }^{2}$, \\ Masatoshi Wakui ${ }^{3}$, Hiromitsu Jinno ${ }^{1}$, Hirotoshi Hasegawa', Shyamala Maheswaran ${ }^{4}$, Makoto Suematsu ${ }^{2}$ \\ and Yuko Kitagawa' ${ }^{1}$
}

\begin{abstract}
Background: Homeobox B9 (HOXB9), a transcriptional factor, regulates developmental processes and tumor progression and has recently been recognized as one of important transcriptional factors related to angiogenesis. This study aimed to investigate the role of HOXB9 in tumorigenesis and angiogenesis.

Methods: We examined the expression of HOXB9 in colorectal cancer using qPCR and in situ hybridization. We also examined the effect of HOXB9 overexpression in colorectal cancer using a proliferation assay, ELISA, a multiplex assay, and xenograft models. The clinical significance of HOXB9 was statistically evaluated in resected specimens.

Results: HOXB9 was expressed in colorectal cancer specimens. HOXB9 induced angiogenesis and tumor proliferation in vitro, which resulted in high tumorigenicity in vivo and poor overall survival. Bevacizumab, an anti-vascular endothelial growth factor (VEGF) antibody, remarkably suppressed tumor proliferation by inhibiting angiogenesis in HOXB9-overexpressing xenografts, and it improved overall survival and provided prolonged progression-free survival in HOXB9-overexpressing patients. A comprehensive multiplex assay of the supernatant of cancer cells co-cultured with human vascular endothelial cells and fibroblasts indicated significantly higher interleukin-6 (IL6) levels than those in the supernatant of monocultured cells. HOXB9 overexpression in clinical specimens was significantly correlated with increased IL6 expression. An IL6-neutralizing antibody inhibited VEGF secretion and tumor proliferation in the co-culture system.

Conclusions: HOXB9 promotes the secretion of angiogenic factors, including VEGF, to induce tumor proliferation through microenvironmental production of cytokines including IL6 signaling. Moreover, silencing of VEGF or IL6 terminates cytokine release in tumor microenvironment. Thus, HOXB9 and IL6 may be potential biomarkers for bevacizumab treatment.
\end{abstract}

Keywords: Colorectal cancer, Aangiogenesis, Bevacizumab, Biomarker

\section{Background}

Homeobox (HOX) genes are key regulators of embryonic development and are evolutionarily conserved [1]. Thirtynine HOX genes are arranged in 4 different clusters, that is, HOXA-HOXD. Homeobox B9 (HOXB9) is expressed in several adult human tissues, including the endometrium [2], mammary glands [3], and blood precursor cells

\footnotetext{
* Correspondence: tetsu@z7.keio.jp

${ }^{1}$ Department of Surgery, School of Medicine, Keio University, 35

Shinanomachi, Shinjuku-ku, Tokyo 160-8582, Japan

Full list of author information is available at the end of the article
}

[4]. HOXB9 has been shown to be overexpressed in human breast cancer and is associated with tumorigenicity, lung metastasis, and radioresistance [5,6]. Especially, HOXB9 induced tumor proliferation and metastasis by activating angiogenesis [5], but little is known about the relationship between HOXB9 expression and angiogenesis in colorectal cancer, which is widely treated by antiangiogenic therapy in combination with chemotherapy, and about the impact of anti-angiogenic treatment on tumors with angiogenic properties.

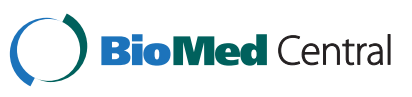


Angiogenesis plays critical roles in tumor growth $[7,8]$ and cancer metastasis [9] because the generation of new vessels to meet the metabolic demands of under-perfused regions promotes tumor growth. Vascular network recruitment provides the principal route by which tumor cells exit the primary tumor site and enter the circulation. Thus, angiogenesis has become a target for cancer therapy through inhibition of vascular endothelial growth factor (VEGF) signaling by various drugs, including bevacizumab, sorafenib, and sunitinib.

Bevacizumab is a humanized monoclonal antibody that inhibits VEGF activity. Because VEGF stimulates the angiogenesis of microvessels, bevacizumab is proposed to function by blocking neovascularization in the tumor microenvironment $[10,11]$ and by reducing vascular permeability in tumor microvessels [12,13]. Bevacizumab has become one of the standard components of chemotherapy for metastatic or recurrent colorectal cancer, but no biomarkers are available to determine the efficacy of anti-VEGF therapy.

It is important to investigate the mechanisms underlying anti-angiogenic treatment, especially in highly vascular tumors. Here, the clinical significance of HOXB9 expression was assessed, and the angiogenic and oncogenic functions of HOXB9 were further examined in vivo and in vitro. In the present study, we have demonstrated that anti-angiogenic treatment inhibits tumor proliferation driven by HOXB9 overexpression and decreases the tumor burden by silencing cytokine crosstalk in microenvironment.

\section{Results}

HOXB9 expression in colorectal cancer patients

To investigate HOXB9 expression in colorectal cancer, we examined specimens from patients who had p-stage II or III colorectal cancer and had undergone surgery at our institute. HOXB9 mRNA was expressed in approximately $70 \%$ of the patients (Figure 1a), and HOXB9 was found to be upregulated in a fraction of tumors, relative to that in the uninvolved adjacent normal mucosa, in both RNA in situ hybridization (Additional file 1: Figure S1a), and immunohistochemical staining (Figure 1b). HOXB9 mRNA expression was significantly higher in poorly differentiated adenocarcinomas than in highly and moderately differentiated types of adenocarcinomas (Figure 1c) and was highly related to liver metastasis (Additional file 1: Figure S1b). Moreover, although HOXB9 did not affect the disease free survival after surgery, increased HOXB9 expression in colorectal cancer was significantly associated with a poor prognosis in overall survival (Figure 1d), as previously reported for breast cancer [14].

\section{Role of HOXB9 in angiogenesis, TGF-beta axis and tumorigenesis}

We introduced the HOXB9 construct into the HT29 and HCT116 cancer cell lines (HT29-T and HCT116-T, respectively), both of which showed low HOXB9 mRNA expression relative to that in the other human colorectal cancer cell lines (Additional file 1: Figure S1c), in order to evaluate the functional consequence of HOXB9 overexpression in colorectal cancer. HOXB9 overexpression is regulated by a tetracycline repressor (Figure 1e,f and Additional file 1: Figure S1d,e) [15]. Angiogenic factors, including amphiregulin (AREG), epiregulin (ERG), basic fibroblast growth factor (bFGF), interleukin-8 (IL8), and VEGF were significantly upregulated by HOXB9 (Figure 1g). To determine the effect of the loss of HOXB9, we produced short hairpin RNA (shRNA)expressing HOXB9 lentiviral constructs capable of reducing endogenous HOXB9 expression by up to $99 \%$ (Figure 1e). These constructs were used to reduce the expression of angiogenic factors in WiDr and SW480 cell lines (WiDr-S and SW480-S, respectively) that initially had high expression of HOXB9 (Figure 1g). Moreover, the transforming growth factor (TGF) -beta axis was regulated by HOXB9 in colorectal cancer (Figure 1h), similar to the results previously obtained for breast cancer [5]. Next, we investigated the functional significance of these factors in ELISA assays. Significantly increased tumoral secretion of angiogenic factors were confirmed in the supernatants of HOXB9-overexpressing cell lines (Additional file 2: Figure S2a,b). To confirm the role of this acquired angiogenesis of colorectal cancer in vivo, we established human tumor xenografts in BALB/c nude mice. HOXB9-overexpressing xenografts of the HT29-T cell line demonstrated a dramatic increase in tumor burden (Figure 2a,b) and microvessel density (Figure 2c,d and Additional file 2: Figure S2c). The immunohistochemical staining showed higher production of angiogenic factors including IL8 and VEGF in HOXB9 overexpressed tumor (Additional file 2: Figure S2d).

\section{Inhibition of angiogenesis in HOXB9-overexpressing tumors}

The cell proliferation test for 24 hours revealed almost the same proliferation in HOXB9 overexpressed cells as control (Additional file 3: Figure S3a), and the cell viability test for 7 days showed rather smaller survival rate in HOXB9 overexpressed cells in vitro (Additional file 3: Figure S3b). Therefore, the role of the microenvironment in tumor proliferation under the regulation of HOXB9 was investigated, given that the primary difference between the in vivo and in vitro conditions was the existence of a microenvironment including vessels and fibroblasts. When HUVECs and human dermal fibroblasts (HDFs) were co-cultured with cancer cell lines to 


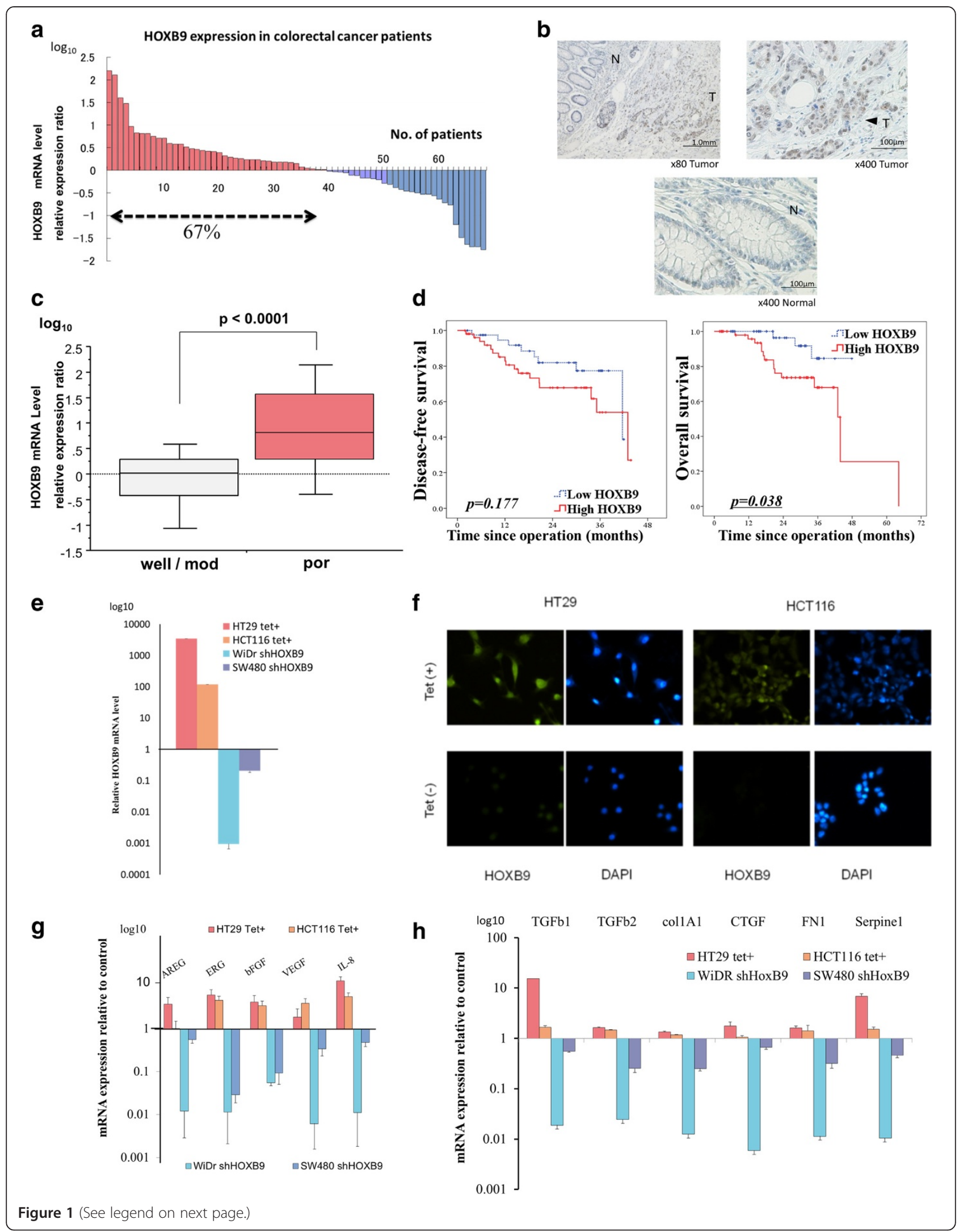


(See figure on previous page.)

Figure 1 HOXB9 expression in colorectal cancer. (a) Waterfall plot of relative mRNA expression levels of HOXB9 in patients at the cancer site compared with those in normal mucosa $(n=69)$, determined using qRT-PCR in triplicate. (b) Immunohistochemical staining of HOXB9 in resected colorectal cancer specimens ( $T$, tumor; N, normal mucosa). (c) Relative mRNA expression of HOXB9 in patients according to pathological differentiation (Well/ mod, well or moderately differentiated adenocarcinoma; Por, poorly differentiated adenocarcinoma). (d) Kaplan-Meier curves of colorectal cancer patients ( $n=93$ ), according to HOXB mRNA expression (Left panel, disease free survival; right panel, overall survival). (e) mRNA expression of HOXB9 determined by RT-PCR. (f) Immunocytochemistry of HOXB9-overexpressing cell lines. (g and $\mathbf{h}$ ) mRNA expression of angiogenic factors (g) and TGF beta family (h) in HOXB9-overexpressing cell lines using RT-qPCR in triplicate. Error bars are SDs. P values by the log rank test. Scale bars, 100 um.

mimic the microenvironment (Additional file 3: Figure S3c,d), HOXB9 significantly promoted cell proliferation (Figure 3a). Then, we hypothesized that anti-angiogenic treatment may cause strong regression in tumors with such angiogenic properties as HOXB9, which depends tumor proliferation on enhanced angiogenesis and stromal reaction in the microenvironment. In order to evaluate the impact of anti-angiogenic agents on tumor microenvironment in vitro, the tube formation of HUVECs co-cultured with HOXB9-regulated cancer cells was estimated under administration of bevacizumab. HOXB9 significantly enhanced angiogenesis, and bevacizumab administration significantly reduced angiogenesis of HUVECs co-cultured with HOXB9-overexpressing cancer cells (Figure 3b and Additional file 3: Figure S3e,f).

Next, BALB/c nude mice transplanted with xenografts that had been previously established using HOXB9overexpressing colon cancer cells were treated intraperitoneally with bevacizumab (5 $\mathrm{mg} / \mathrm{kg}$, weekly). Both of HOXB9-induced cancer cells showed accelerated tumorigenesis. However, the bevacizumab treatment dramatically reduced tumor volume and tumor weight both in HT29-T cells (Figure 4a,b) and in HCT116-T (Additional file 4: Figure S4a,b). Tumor gross vascularity was also reduced by bevecizumab (Figure 4c). The growth inhibition mediated by bevacizumab significantly increased in HOXB9overexpressing tumors (Figure 4d,e and Additional file 4: Figure S4c,d). We further established an intrasplenic injection (ISP) model of HCT116-T cells in BALB/c nude mice to determine whether anti-angiogenic treatment with bevacizumab prolongs survival in angiogenic tumors. HOXB9 overexpression was associated with longer third-quadrant survival upon bevacizumab treatment (Additional file 5: Figure S5a,b). To vertify our hypothesis that the loss of angiogenic factors plays an important role in tumor shrinkage in angiogenesis-driven tumor, sunitinib malate, which is a multiple tyrosine kinase inhibitor with effects including VEGF inhibition, was also administered in HOXB9 overexpressed tumor. Sunitinib also suppressed cell proliferation in HOXB9-overexpressing breast cancer xenografts (Additional file 5: Figure S5c).

\section{Enhanced cytokine release from microenvironment}

To determine the key factors for enhanced tumor growth in microenvironment, we performed comprehensive multiplex assays involving the co-culture supernatants and observed significant elevation of the levels of several cytokines, including interleukin-6 (IL6) (Figure 5a). We then focused on the differences between the secretion of IL6 in monoculture and co-culture systems to elucidate the impact of microenvironment on tumor proliferation. The concentration of IL6 was dramatically higher in the co-culture system than in the mono-cultures (Figure $5 \mathrm{~b}$ and Additional file 6: Figure S6a), suggesting enhanced IL6 secretion in microenvironment including tumor and stromal cells. To confirm the importance of microenvironmental IL6 secretion, cell proliferation in the presence of an IL6 neutralizing antibody was evaluated. We also used bevacizumab to inhibit the activity of VEGF released from cancer cells (Figure 5c). Both the IL6 neutralizing antibody and bevacizumab produced significant reduction of cancer cell proliferation (Figure 5d,e and Additional file 6: Figure S6b). Moreover, IL6 secretion in the supernatant dramatically decreased on administration of bevacizumab in co-culture systems (Figure 5f).

\section{Clinical significance of HOXB9 on bevacizumab treatment}

To clarify the clinical significance of anti-angiogenic treatment for angiogenic colorectal cancer, we conducted a retrospective study of patients who had undergone surgery between 2001 and 2010 at our institute and were treated by chemotherapy, including bevacizumab $(n=39)$. Specimens obtained from patients before chemotherapy were analyzed by Quantitative reverse transcription-PCR (qRT-PCR). The demographic and clinical data showed no significant differences with regard to age, sex, pathological stage, or chemotherapy between groups with high $(\mathrm{n}=13)$ or low $(n=26)$ expression of HOXB9 (Additional file 7 : Table S1). High expression of HOXB9 mRNA was significantly associated with longer overall survival and progression-free survival after treatment with bevacizumab combined with chemotherapy (Figure 6a,b), and mRNA expression of HOXB9 was significantly correlated with IL6 mRNA expression in clinical specimen (Figure 6c).

\section{Discussion}

HOXB9 has been reported to be overexpressed in neoplastic tissues [16]; to promote the expression of angiogenic factors, ErbB, and TGF-beta ligands; and 

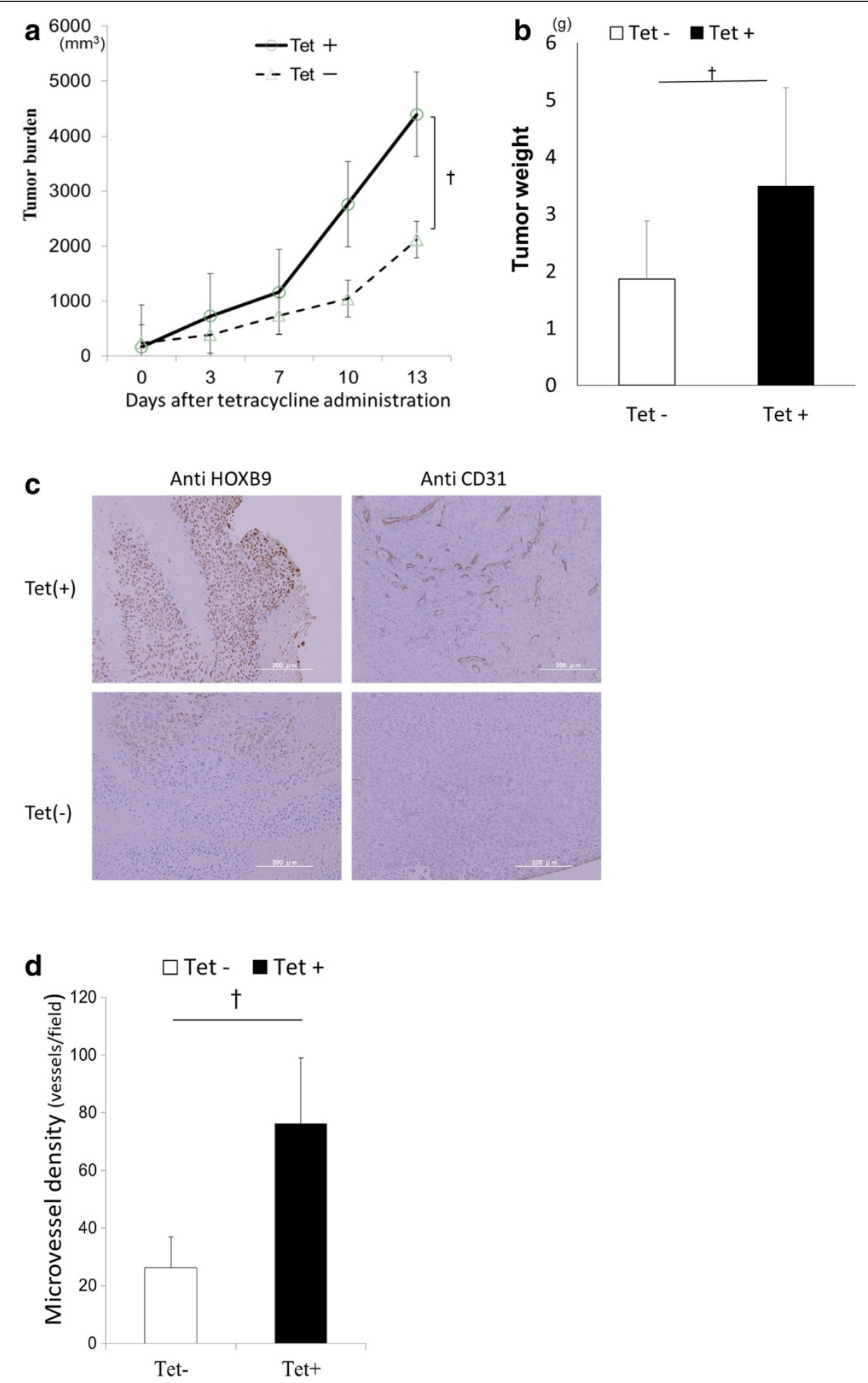

Figure 2 Impact of HOXB9 expression on tumor xenografts. (a, b) Subcutaneous xenograft model of HT 29-T cells was established in Balb/c nu/nu mice ( $n=8$, each). Tumor burden (a) and tumor weight (b) were evaluated. (c) Immunohistochemical staining of paraffin-embedded xenografts obtained from HT29-T cancer cells. Samples were stained in anti-HOXB9 or anti-CD31. (d) Microvessel density of xenograft tumor was evaluated and measured in immunohistochemical staining by ant-CD31 antibody in xenografts ( $n=8$, each). Error bars are SDs. $\star p<0.05$ and $+p<0.005$ by U-test. Scale bars, $200 \mu \mathrm{m}$. 


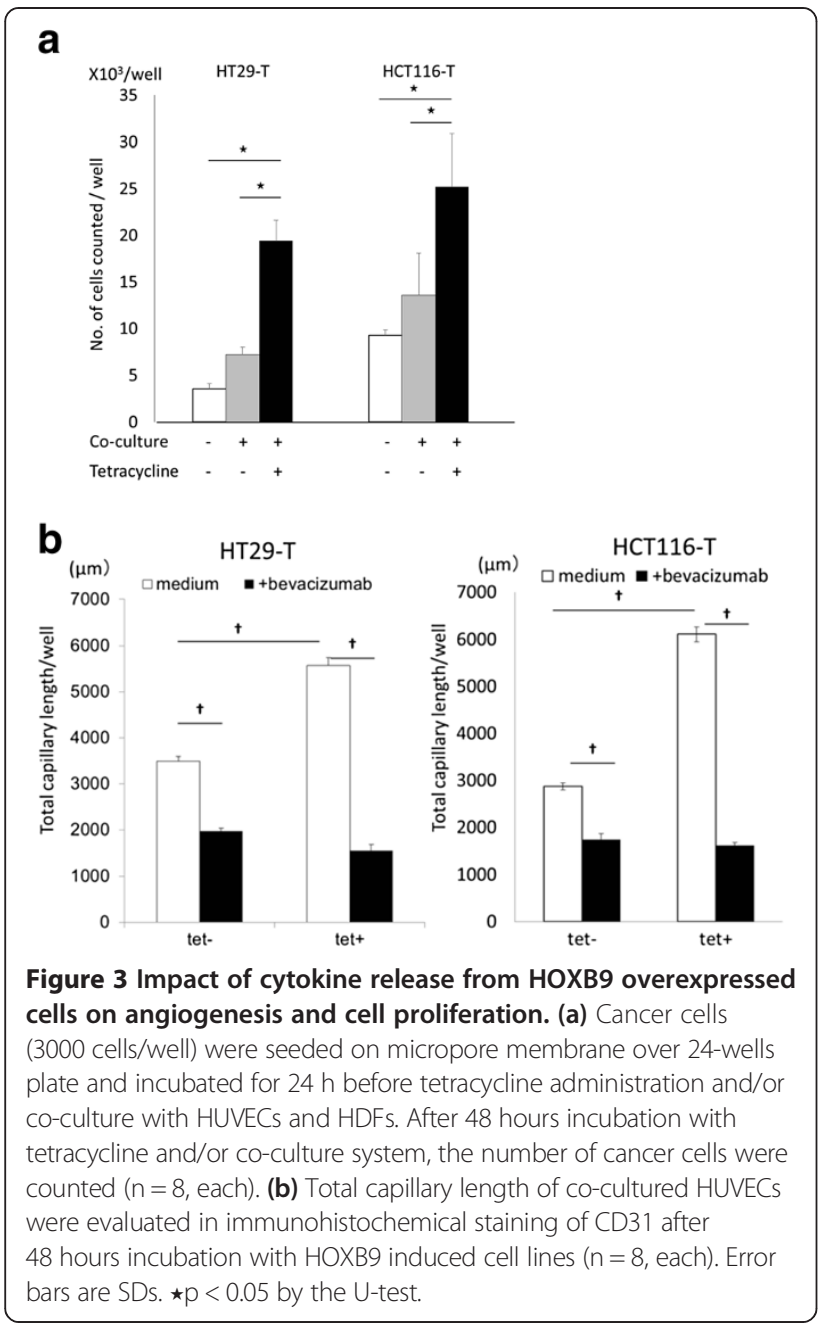

to promote angiogenesis and distant metastasis in breast cancer [5]. From a biological perspective, multiple HOX-binding sites have already been identified in the promoters of angiopoietin-like 2, IL8, TGF-beta2, VEGF, and bFGF [17]. AREG, ERG, VEGF, and bFGF were previously revealed to be the direct targets of HOXB9 [5,18]. In bioinformatic analysis using PROMO3.0 (http://alggen.lsi. upc.es/cgi-bin/promo_v3/promo/promoinit.cgi?dirDB= TF_8.3), we found multiple sequences in the promoter regions of IL8 where HOX genes were predicted to bind. In the present study, we demonstrated that HOXB9 also promoted the expression of angiogenic factors and TGFbeta ligands in colorectal cancer. Tumorigenesis was enhanced by the microenvironment, as demonstrated by stromal interaction and neovascularization in HOXB9overexpressing tumors in vivo. In addition to angiogenesis, the stromal response has been suggested as one of the determinants of tumor proliferation and recurrence in colorectal cancer [19]. The tumor stroma is activated by TGF-beta signaling, which leads to upregulation of extracellular matrix proteins and inflammatory, angiogenic, and invasion factors that promote the growth of tumor cells $[20,21]$. A study involving chromatin immunoprecipitation previously demonstrated that HOXB9 binds to the TGF-beta2 promoter region as a transcriptional factor to regulate gene expression [5]. Taken together, the activation of angiogenesis and TGF-beta axis in vitro may result in strong tumorigenesis in vivo and poor overall survival in colorectal cancer patients.

To vertify our hypothesis that angiogenic tumors which depend the tumorigenity on angiogenesis are more sensitive to anti-angiogenic treatment, the present study has demonstrated three important findings: the relationship of HOXB9 to colorectal cancer tumorigenicity in vitro and in vivo in terms of angiogenesis and TGF beta signaling; the association of VEGF and antiangiogenic agents with the tumorigenic HOXB9 effect; and, the association of HOXB9 with accelerated cytokine release from tumor microenvironment. For the investigation of tumor suppression by bevacizumab administration, we performed the multiplex assay for comprehensive detection of enhanced cytokine production induced by HOXB9 in co-culture experiments. Our study which compared co-culture with mono-culture demonstrated that tumorigenic effects of HOXB9 in the microenvironment resulted in the enhanced release of various cytokines and that IL6 release from tumor microenvironment plays an important role in tumor proliferation. Not only silencing of IL6 led to the reduction in the proliferation of tumor in co-culture experiments, but also bevacizumab administration reduced IL6 release itself from tumor microenvironment. Thus, these on-and-off results for HOXB9 expression and neutralizing antibodies suggest our hypothesis that bevacizumab inhibits HOXB9 induced tumor proliferation by silencing microenvironmental cytokine release including VEGF and IL6 (Additional file 6: Figure S6c). The microenvironment in humans includes not only vessels and fibroblasts but also immune systems, which results in complicated interactions. In the present study, the predominance of cytokine secretion between tumor and stroma is not evident, and a role for IL6 as another mediator cannot be excluded. But our data suggest a possible role for microenvironmental IL6 in tumor cell proliferation, at least with regard to angiogenesis.

Our results are consistent with those of previous studies demonstrating that IL6 affects microenvironment. IL6 levels are known to be increased in most epithelial tumors [22], and high serum IL6 levels are associated with poor clinical outcomes in patients with colorectal cancer [23] or ovarian cancer [24]. IL6 is a key mediator in a mouse model of microbially induced colorectal cancer [25], possibly through induction of cancer-related molecular pathways such as those involving STAT3 [26].

Several limitations affect the interpretation of the present findings. First, this translational study was based 

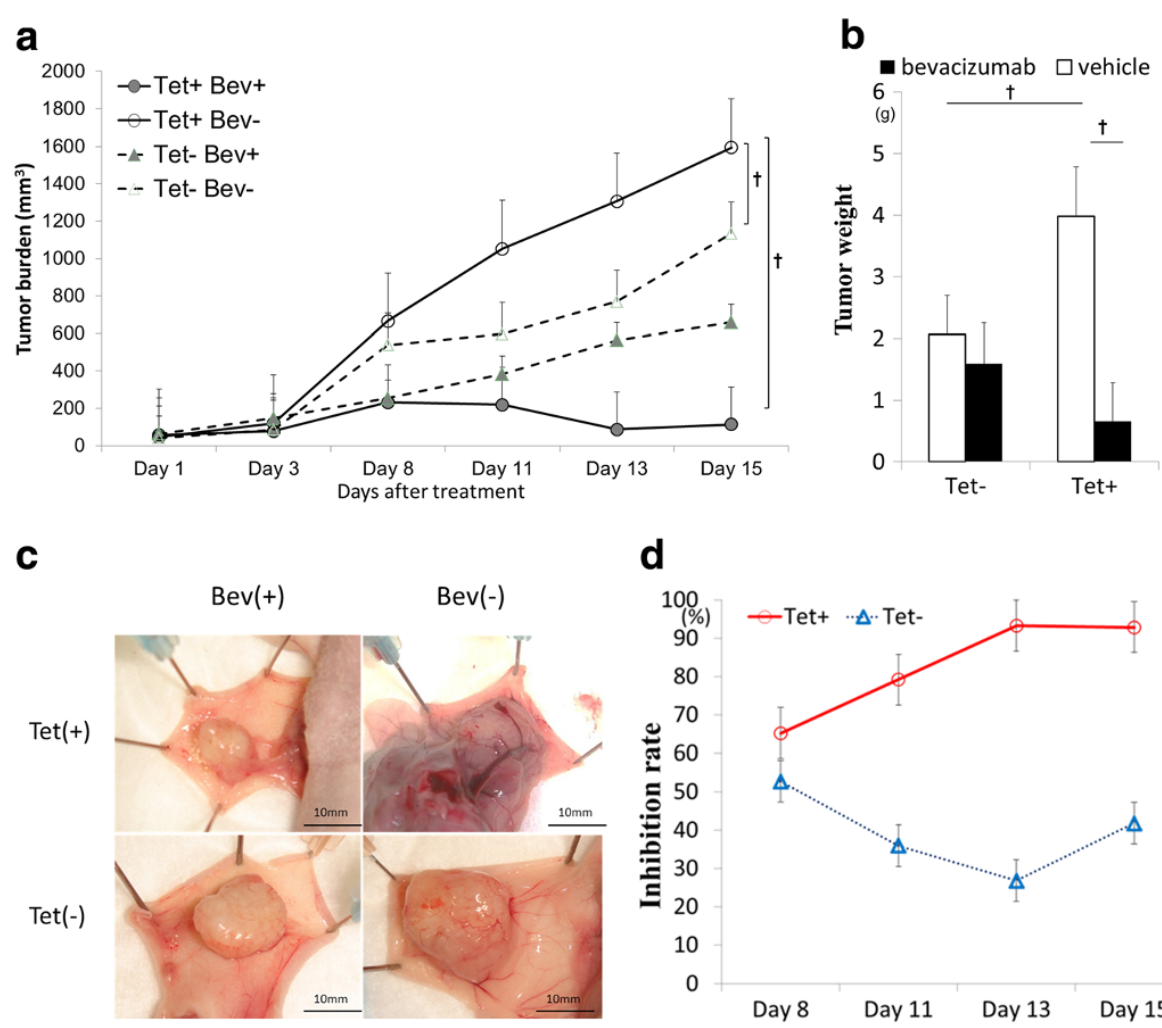

d
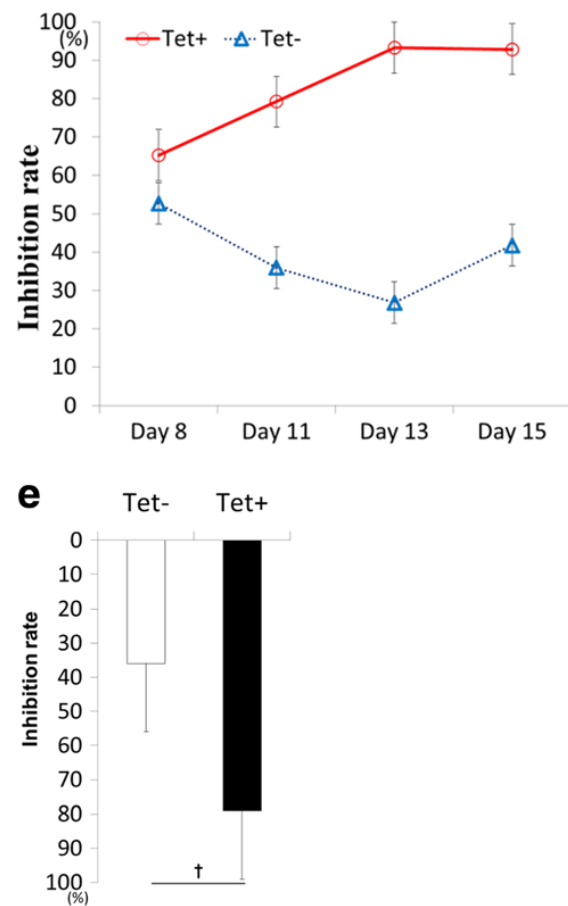

Figure 4 Bevacizumab treatment and HOXB9 expression. Subcutaneous xenografts of HT 29-T cells in Balb/c nu/nu mice were treated with bevacizumab ( $n=8$, each). Tumor growth under bevacizumab treatment were evaluated in tumor burden (a), tumor weight (b), tumor specimen (c), and tumor inhibition rate (d). (e) Tumor inhibition rate was calculated in HT 29-T on day 13. Error bars are SDs. $\star p<0.05$ and $+p<0.005$ by U-test.

on a retrospective analysis in a single center and a small number of patients were included. Second, the selection of patients for the bevacizumab treatment was subjective. The clinical significance of HOXB9 and IL6 must therefore be addressed in a prospectively planned multicenter trial. Multigene assays involving a large number of specimens may provide more reliable insights into tumor biology and the response to bevacizumab regimens. Third, the relationship between the immunological response and chemotherapy combined with antiangiogenic treatment is unclear because bevacizumab was not administered as a single agent in our clinical study. Fourth, the impact of a combined cytotoxic chemotherapy regimen is unclear; the results obtained in the present study are biased by the chemotherapy itself.

\section{Conclusions}

HOXB9 is also expressed in colorectal cancer and functions as a strong tumorigenic factor through activation of the TGF-beta axis and angiogenesis. Colorectal cancer patients with HOXB9 overexpression had poor overall survival in this study, but conversely demonstrated better overall survival with bevacizumab treatment. Bevacizumab suppressed secretion of IL6 into the microenvironment 


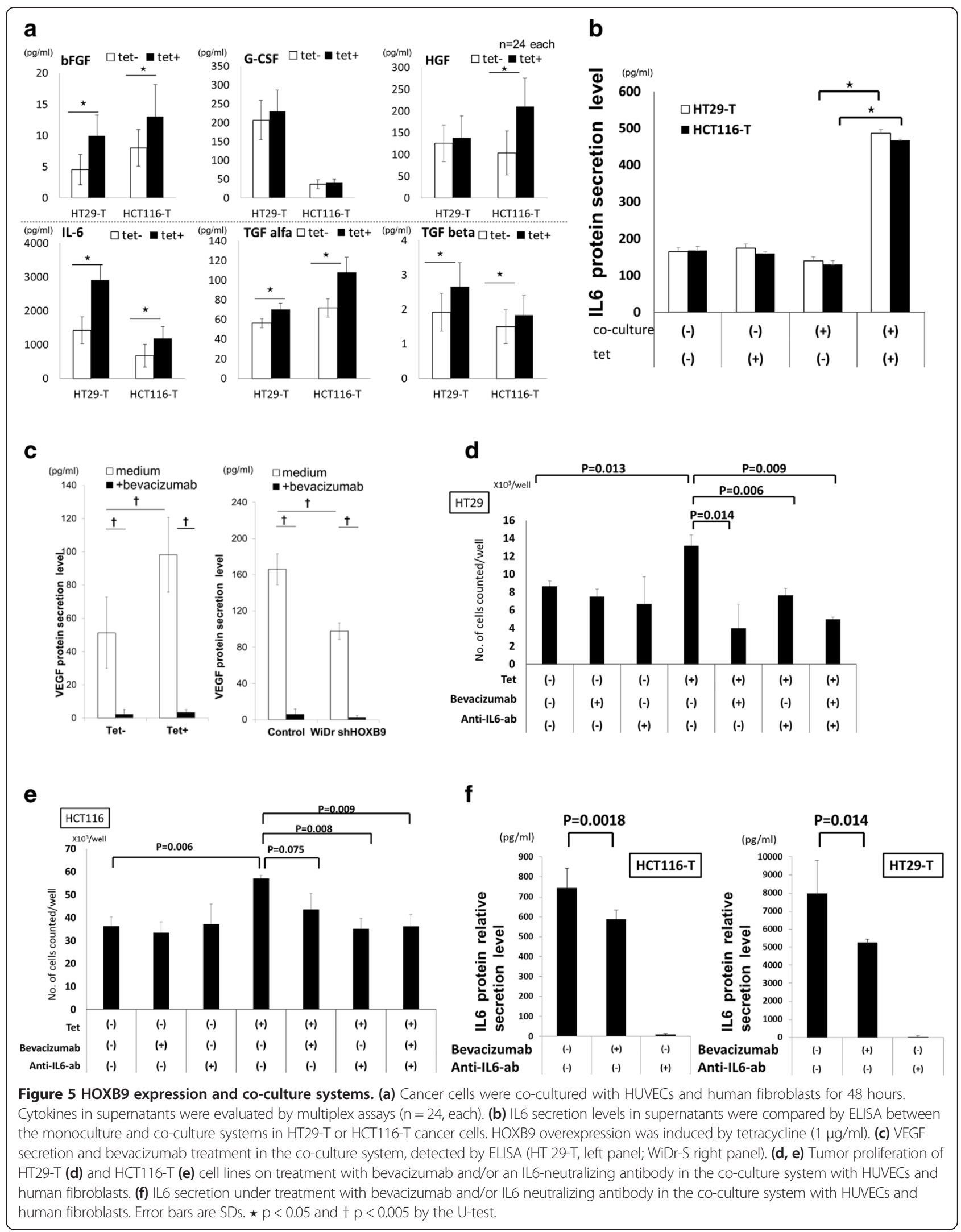




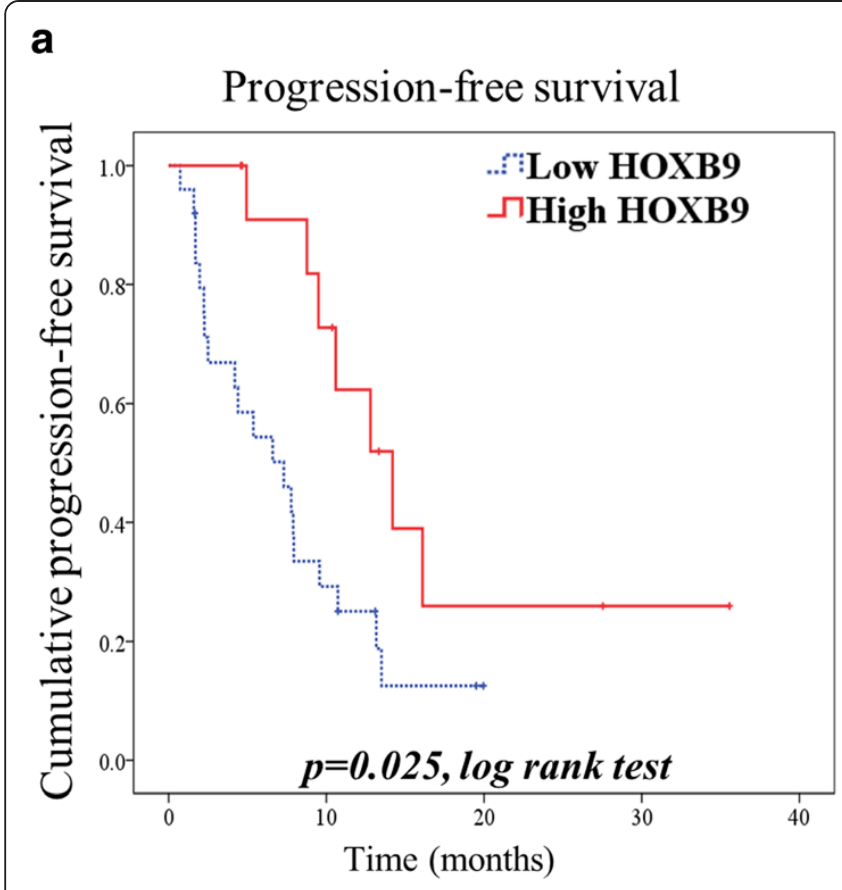

b
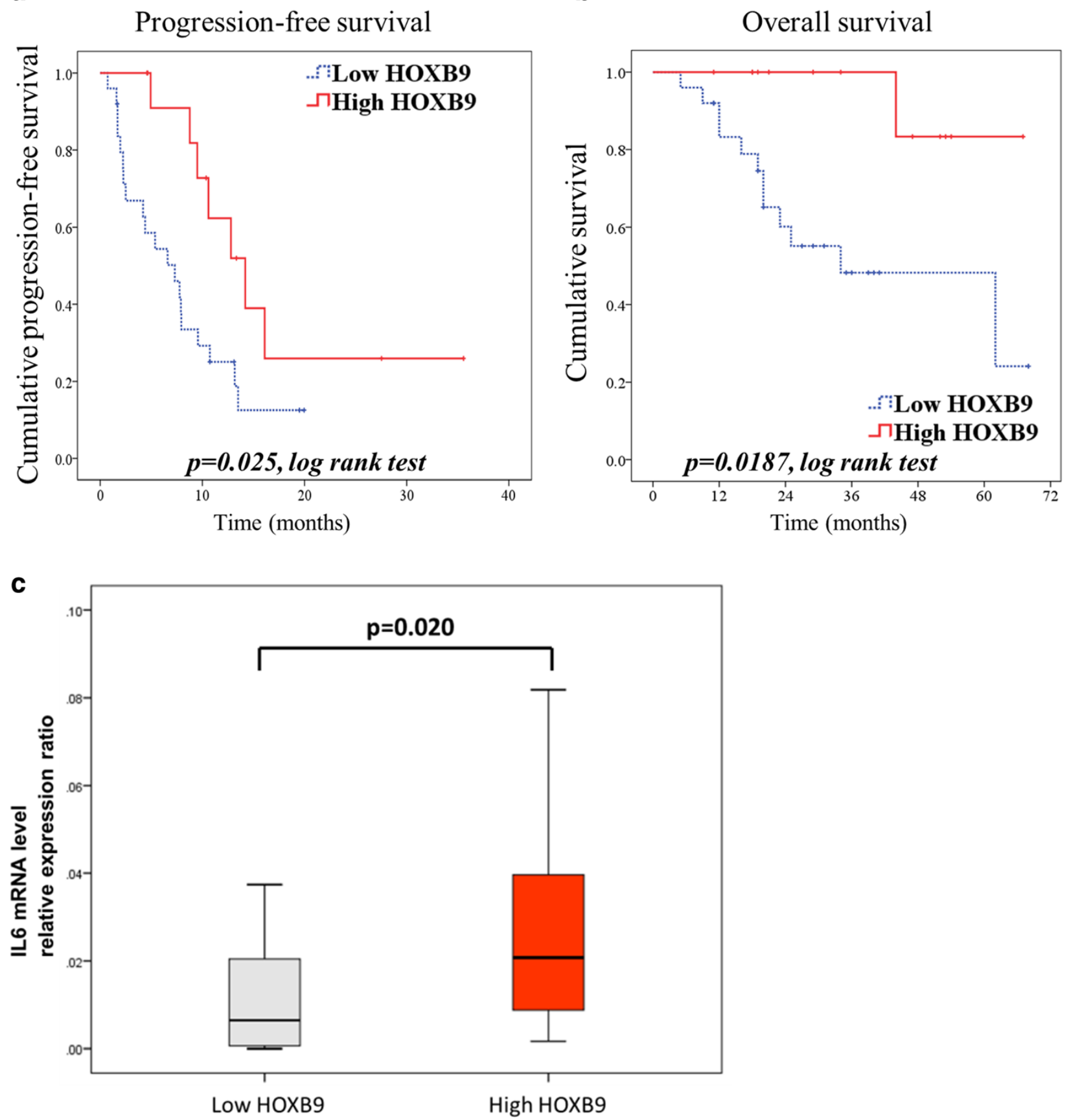

Figure 6 Clinical data for colorectal cancer patients undergoing bevacizumab treatment. HOXB9 mRNA expression levels were evaluated in triplicate by qRT-PCR in patients who showed recurrence after surgery and were treated by chemotherapy combined with bevacizumab ( $\mathbf{a}$ and $\mathbf{b}$ ) Kaplan-Meier curves of colorectal cancer patients, according to HOXB mRNA expression. Progression-free survival (a) and overall survival (b) $\mathrm{p}$ values by the log rank test. (c) IL6 mRNA expression in colorectal cancer specimens. Error bars are SDs. P values by the U-test and log rank test.

in vitro, which suggests a possible mechanism for the increased response to bevacizumab treatment. Previous studies have reported the clinical impact of some genes, including VEGF splice isoforms or neuropilin-1 (NRP-1) [27]. However, to date, no biomarkers have been identified in randomized controlled trials on the clinical response of anti-angiogenic treatments in colorectal cancer [28]. Further prospective studies on HOXB9 and IL6 in different specimens such as serum samples are important to clarify the utility of these molecules as biomarkers. 


\section{Methods mRNA expression}

Our analysis included colorectal tumors of patients who presented between January 2004 and January 2008 at Keio University Hospital; these data were obtained from the hospital colorectal cancer database. Among the samples of consecutive sporadic colorectal cancer patients who received a diagnosis of p-stages II or III disease, 69 panels of frozen tissue specimens containing cancerous and matched normal mucosal tissue were available for qRT-PCR assessment. All samples were obtained from surgically resected material, immediately frozen in liquid nitrogen, and stored at $-80^{\circ} \mathrm{C}$. Adequate lymph node dissection was performed in all these cases, and a pathological diagnosis was made according to the TNM classification. HOXB9 mRNA expression was examined at cancer sites and in normal mucosa in consecutive pStages II and III patients who had undergone surgery at our institute during 2004-2008. The expression ratios (cancer site to normal mucosa) were examined using qRT-PCR. RNA was extracted from the cells by using the RNeasy kit (QIAGEN, Valencia, CA). Conditions for semiquantitative amplification of cDNA were $95^{\circ} \mathrm{C}$ for $2 \mathrm{~min}$, followed by 25 cycles of $95^{\circ} \mathrm{C}$ for $30 \mathrm{~s}, 56^{\circ} \mathrm{C}$ for $30 \mathrm{~s}$, and $72^{\circ} \mathrm{C}$ for $60 \mathrm{~s}$, with a final extension cycle of $72^{\circ} \mathrm{C}$ for $10 \mathrm{~min}$. RT-PCR analysis was run in triplicate for each sample on a Light Cycler 480 Real-Time PCR System using SYBR Green 1 Master Mix (Roche). The following program was run: preincubation for $5 \mathrm{~min}$ at $95^{\circ} \mathrm{C}$, amplification for 45 cycles $(10 \mathrm{~s}$ of denaturation at $95^{\circ} \mathrm{C}, 10 \mathrm{~s}$ of annealing at $57^{\circ} \mathrm{C}$, and a 10 -s extension at $72^{\circ} \mathrm{C}$ ), and melt-curve analysis. mRNA levels of the target gene were normalized against mRNA levels of GAPDH, which was used as an internal control. The sequences of all the primers used are listed in Additional file 8: Table S2. Written informed consent was taken and the study was approved by the ethics committee of the School of Medicine of Keio University.

\section{In situ hybridization}

The HOXB9 probe for ISH was generated as described previously [5]. Briefly, the plasmid encoding HOXB9 was cleaved with Hinc II and XmaI (New England Biolabs). The acquired 286-bp fragment was outside the homeodomain and spanned nucleotides 26-324 of the HOXB9 transcript. This region had 100\% homology to HOXB9, as expected, and had little homology with other members of the HOX family or other HOX genes. After linearization of the plasmid, in vitro transcription was carried out, using 11-digoxogem-UTP (Roche) and 2 units/ $\mu \mathrm{L}$ of either T3 of T7 RNA polymerase (Promega) for sense and antisense probes, respectively. Riboprobes were purified by ethanol precipitation, and their size and integrity checked by gel electrophoresis. The tissues were dehydrated, washed twice with PBS, and treated with 3\% hydrogen peroxide for $15 \mathrm{~min}$. Following treatment with proteinase $\mathrm{K}(10 \mu \mathrm{g} / \mathrm{ml})$ for $9 \mathrm{~min}$, tissues were washed with PBS and refixed with $4 \%$ paraformaldehyde in PBS for $5 \mathrm{~min}$. The tissues were pre-hybridized for $1 \mathrm{hr}$ at $65^{\circ} \mathrm{C}$ before hybridizing with sense or anti-sense digoxigenin-labeled riboprobes overnight at $70^{\circ} \mathrm{C}$. After hybridization, samples were placed in 1\% sheep serum for $30 \mathrm{~min}$ at room temperature, and then incubated with anti-digoxygenin-AP (Roche) antibody for 2 hours at room temperature. BM-Purple precipitation (Roche) was used to detect the signal.

\section{Cell lines and cell culture}

The human colorectal cancer cell lines SW480, WiDr, HT-29, and HCT116 were obtained from the American Type Culture Collection (Rockville, MD). The cell lines HT29 and HCT116 were seeded at a density of $1 \times 10^{6} / \mathrm{ml}$ in RPMI 1640 medium (Gibco, MA), and SW480 and WiDr cells were seeded a density of $1 \times 10^{6} / \mathrm{ml}$ in Dulbecco's modified Eagle medium. Both media were supplemented with 10\% FBS (CSL Ltd., Australia), $100 \mathrm{IU} / \mathrm{ml}$ penicillin, and $50 \mathrm{mg} / \mathrm{ml}$ streptomycin sulfate. HT29 and HCT116 cells with low HOXB9 expression were infected with HOXB9-expressing lentiviral constructs (Invitrogen). For the Tet-regulated system, the entry vector was transferred to the lentiviral pLenti4/TO/ V5-DEST vector and used in combination with the Tetrepressor vector pLenti6/TR (Invitrogen) according to the manufacturer's instructions. The induction of HOXB9 was regulated by the addition of $1 \mu \mathrm{g} / \mathrm{ml}$ tetracycline (Invitrogen). To knock down HOXB9 expression, viral constructs of shRNA were generated, and SW480 and WiDr cells were infected as described previously [5].

\section{Edu cell proliferation assay}

DNA synthesis was measured using 5-ethynyl-2'-deoxyuridine (EdU) Flow Cytometry Assay Kits (Life Technologies, Tokyo, Japan). Experiments were performed according to the manufacturer's instructions. Briefly, $3 \times 10^{6}$ cells were seeded into $10 \mathrm{~cm}$ dish and incubated for 24 hours at $37^{\circ} \mathrm{C}$ with or without $1 \mu \mathrm{g} / \mathrm{ml}$ tetracycline (Invitrogen). Cells were incubated for 2 hours with $20 \mu \mathrm{M}$ EdU. Subsequently, cells were processed using the Click-iT EdU Flow Cytometry kit. EdU incorporation was measured by Flow cytometer (Gallios Flow Cytometer, Beckman Coulter).

\section{MTT cell proliferation assay}

Cell proliferation was measured using the 2,3-bis (2methoxy-4-nitro-5-sulfophenyl)-2H-tetrazolium-5-carboxanilide inner salt (MTT) assay. Briefly, 5,000 cells per well in 96-well microtiter plates (Sumilon, Sumitomo Bakelite Co., Tokyo, Japan) were incubated for $24 \mathrm{~h}$, followed by continuous exposure to $1 \mu \mathrm{g} / \mathrm{ml}$ tetracycline 
(Invitrogen) for 6 days. The absorbance in the wells was measured at every 2 days using a NJ-2300 microplate spectrophotometer at 540 and $630 \mathrm{~nm}$ (Immuno Reader, Nalge Nunc International, Rochester, NY).

\section{Western Blotting, Immunocytochemistry, and immunohistochemistry}

Expression of proteins were detected in western blotting using antibody against HOXB9 (Santa Cruz Biotechonlogies), pAKT (Cell Signaling Technology), AKT (Cell Signaling Technology), VEGF (ABCAM) and GAPDH (ABCAM). The western blot protocols used have been described previously [29]. For immunocytochemistry, cells were plated in 8-well chamber slides (Lab Tek) and treated as described previously [5]. Briefly, after blocking, the cells were incubated with an anti-human HOXB9-specific antibody (rabbit polyclonal antibody; Santa Cruz Biotechnology, Santa Cruz, CA) at a dilution of 1:250 or an anti-CD31 antibody (ab28364; ABCAM) at 1:200 for $1 \mathrm{~h}$ at room temperature in incubation buffer (phosphate-buffered saline (PBS) containing 1\% BSA). For immunohistochemistry, HOXB9, CD31, CD34, IL8, and VEGF expression was detected using the anti-human HOXB9-specific antibody at a dilution of 1:250 (Santa Cruz, CA), the anti-CD31 antibody at a dilution of 1:200 (ab28364; ABCAM), the antiCD34 antibody at a dilution of 1:200 (ab8158; ABCAM), anti-IL8 antibody at dilution of 1:1000 (ab7747; ABCAM), the anti-VEGF antibody at a dilution of 1:1000 (ab46154; ABCAM).

\section{Angiogenesis in vitro and co-culture systems}

Angiogenesis was evaluated based on the microvessel density of HUVEC cultures. Culture supernatants without FBS or antibiotic supplementation were assessed using an angiogenesis kit (Kurabo, Okayama, Japan). Experiments were performed according to the manufacturer's instructions. The angiogenesis kit consisted of a 24-well cluster dish in which HUVECs and fibroblasts were seeded under optimal conditions for capillary tube formation. The microvessel density was calculated as the sum of the total capillary length in 6 areas at $200 \times$ magnification by using the Image J software $(\mathrm{NIH}$, Bethesda, MD). HUVECs and fibroblasts were co-cultured with HOXB9-overexpressing cancer cells and separated from the cancer cells by using micropore membranes. Each well was cultured with serum-free medium corresponding to each cell line, respectively.

Tetracycline was administered to regulate HOXB9 induction after 24 hours of co-culture. Endogenous IL6 was neutralized by application of an anti-IL6 antibody, and VEGF neutralization was assayed in a co-culture system in which $5 \times 10^{3}$ HOXB9-overexpressing cancer cells were seeded per well in a medium supplemented with $1 \mu \mathrm{g} / \mathrm{ml}$ anti-human IL6 antibody (R\&D Systems) and/or $1 \mu \mathrm{g} / \mathrm{ml}$ bevacizumab. HOXB9 expression was controlled by the addition of tetracycline.

\section{Multiplex assay}

Cytokine levels in cell culture supernatants were quantified using ELISA (BD Biosciences Pharmingen for VEGF, HGF, CXCR8, and IL6). Comprehensive proliferative cytokine levels in co- and monoculture supernatants were measured using a Procarta Cytokine Profiling kit (Affymetrix, Santa Clara, CA, USA), which utilizes xMAP multianalyte profiling Luminex technology for simultaneous detection and quantification of multiple protein targets. Fluorescently encoded antibody beads were detected individually in a flow cytometer, and 9 cytokines (bFGF, G-CSF, GMCSF, HGF, IL1beta, IL6, MCSF, TGFbeta, and TGFalpha) were evaluated.

\section{Xenograft experiments}

All the animals were cared for and experiments were performed according to AAALAS guidelines using protocols approved by the institutional review board and the institutional animal care and use committee of Keio University or Massachusetts General Hospital. Six-weekold Balb/c female nude mice were subcutaneously implanted with $4 \times 10^{6}$ cells in $100 \mu \mathrm{l}$ of PBS $(n=8)$. If the tumor size was greater than $75 \mathrm{~mm}^{3}$, oral tetracycline administration was started on day 1 . Tetracycline (SigmaAldrich) was dissolved in distilled water at a concentration of $1 \mathrm{mg} / \mathrm{ml}$. Bevacizumab (5 mg/kg) or PBS was intraperitoneally administered weekly. The tumor volume was calculated from the pi-based ellipsoid volume formula $\pi / 6 \times$ length $\times$ width $\times$ height. Growth inhibition of the tumor was calculated as (1 - (mean tumor volume of treated group/mean tumor volume of vehicle group) $) \times$ 100. An intrasplenic injection (ISP) model was established to create liver metastasis as described previously [30,31]. Briefly, nude mice were anesthetized by ether inhalation, and open laparotomy was performed. HOXB9overexpressing cancer cells $\left(5 \times 10^{6}\right.$ cells of HCT116-T in $100 \mu \mathrm{l}$ of PBS) were injected into the spleen, and splenectomy was then performed. Tumor samples were immunostained using an anti-CD31 antibody (Abcam, Cambridge, UK) and then counterstained with DAPI. Vessels were counted using a microscope in areas of the tumor containing the highest number of capillaries and small venules. Highly vascular areas were first identified by scanning tumor sections, and the vessels were counted in 6 such areas at $200 \times$ magnification. Bevacizumab was purchased from Chugai Pharmaceutical Co. Ltd (Tokyo, Japan). MCF10A cells expressing activated G12V HRasV12-expressing lentivirus and either LacZ or HOXB9 were generated using standard protocols. Xenografts were established by injecting $5 \times 10^{6}$ cells in $100 \mu \mathrm{l}$ of PBS into 
the mammary fat pad of 6-week-old female Swiss nu/nu mice. Tumor volumes were measured at regular intervals.

Sunitinib (SU11248; Sutent) was purchased from LC Laboratories. MCF10A cells expressing activated G12V $\mathrm{H}$-Ras-expressing lentivirus and either LacZ or HOXB9 were injected into the mammary fat pads of mice $(n=16)$. After 2 weeks of tumor growth, 8 animals from each group were treated with $40 \mathrm{mg} / \mathrm{kg}$ sunitinib orally every day for up to 14 days. Sunitinib was dissolved in $0.5 \%$ carboxymethyl cellulose. The remaining 8 animals from each group were treated with the vehicle.

\section{Patients and samples}

Our analysis included 456 colorectal tumors of patients who presented between January 2006 and January 2008 at Keio University Hospital; these data were obtained from the hospital colorectal cancer database. Patients who showed recurrence after surgery and were treated by chemotherapy combined with bevacizumab were selected from the database. Among the specimens from 77 consecutive patients, 39 specimens were available and were analyzed by qRT-PCR. The study was approved by the ethics committee of the School of Medicine of Keio University.

\section{Statistical analysis}

Statistical analysis was performed using the SPSS 18.0 statistical software (SPSS, Chicago, IL). Analyses of age, pathologic tumor size and weight, mRNA expressions, and total capillary length were performed using the Mann-Whitney $U$ test. The tumor size, weight, and burden were statistically evaluated at the time of sacrifice in xenograft models. Disease-free survival (DFS) and overall survival (OS) curves were drawn according to Kaplan-Meier estimates and compared by log-rank tests. DFS was defined as the absence of local or regional recurrence, distant metastasis, second primary carcinoma, or death from any cause. The clinicopathologic categorical variables were compared using Fisher's exact test or logistic regression where appropriate. The cutoff value for HOXB9 mRNA expression was determined according to the receiver operating characteristic curve. $\mathrm{P}<0.05$ was defined as significant.

\section{Additional files}

Additional file 1: Figure S1. HOXB9 expression in colorectal cancer is associated with poor prognosis and enhanced angiogenesis. Relative mRNA expression levels HOXB9 in patients at the cancer site compared with those in normal mucosa, determined using qRT-PCR. (a) In situ hybridization HOXB9 in resected colorectalcancer specimens (As, AntiSense). (b) HOXB9 expression of resected specimens and recurrence. (c) mRNA expression of HOXB9 in human colorectal cancer cell lines were evaluated in triplicate in qRT-PCR (d) mRNA expression of HOXB9 determined by RT-PCR. (e) Western blotting of HOXB9 and related proteins. $p$ values by the $U$ test.
Additional file 2: Figure S2. (a, b) Impact of HOXB9 expression on cytokine and cell proliferation in vitro. Cytokine secretion in HOXB9-over expressing cell lines $(n=8)$. CXCR8 (a) and VEGF (b). (c) Immunohistochemical staining of paraffin-embeddedxenografts obtain from HT29-T cancer cells. Samples were stained in anti-HOXB9 or anti-CD34 antibody. (d) Immunohistostaining of xenografts using anti IL8 antibody and anti VEGF antibody. Error bars are SDs. * $p<0.05$ by U-test.

Additional file 3: Figure S3. (a and b) In vitro cell proliferation of HOXB9 regulated cells evaluated in Edu assays (a) and MU assays (b). (a) Cell proliferation for 24 hours after the induction of HOXB9 in HT29-T cells and HCT116-T cells were evaluated in flow cytometry. (b) Cell proliferation and survival for 7 days in four HOXB9 regulated cell lines were examined in MU assays ( $n=8$ each). (c and $\mathbf{d}$ ) Schematic illustration of coculture systems of tumor cells and HUVECs co-culture systems. Tumors were co-cultured with HUVECs and human fibroblast in the same medium via micro-pore membrane with or without tetracycline to regulate HOXB9 expression to mimic the microenvironment (c). Tumors communicated with microenvironmental cells in a paracrine way (d). (e and f) Immunocytochemical stainings of HUVECs in co-culture system with or without bevacizumab. HUVECs co-cultured with HT29-T (e) or WiDr-S (f) were stained using anti CD31 antibody. Error bars are SDs.

Additional file 4: Figure S4. Bevacizumab treatment and HOXB9 expression (a-d) Tumor growth under bevacizumab treatment in HCT 116-T xenograft models in Balb/c flu/flu mice ( $n=8$ each). (a) Tumor burden were calculated every 3-4 days. (b) Tumor weight were measured at the time of sacrifice. (c) The size of specimens were also evaluated at the time of sacrifice. (d) Tumor inhibition rate was calculated in $\mathrm{Ha}$ 116- T on day 21. Error bars are SDs. ${ }^{*} p<0.05$ and tp $<0.005$ by U-test.

Additional file 5: Figure S5. Xenografted animals treated with bevacizumab and sunitinib. (a and b) HOXB9 expression and bevacizumab treatment were evaluated in ISP model of HCT116-T cells in Balb/c mice ( $n=8$ ). Kaplan-Meier curves for each group (a) and third-quadrant survival period for bevacizumab treatment (b) were analyzed in these mice. (c) Tumor growth under sunitinib treatment in an MCF 1OA-RAS xenograft model in Balb/c nu/nu mice $(n=8)$. Error bars are SDs. tp $<0.005$ by U-test.

Additional file 6: Figure S6. HOXB9 expression and co-culture systems in WiDr cells. (a) Cytokines in supernatants of co-cultured cell lines, detected by ELISA (no significant difference, n8 each). (b) Tumor proliferation of WiDr shHOXB9 and scramble cell lines on treatment with bevacizumab andlor an 1L6-neutralizing antibody in the co-culture system. (c) Scheme of the hypothesis in the present study. Error bars are SDs. p values by U-test.

Additional file 7: Table S1. Consecutive colorectal cancer patients who received bevacizumab treatment after tumor resection were enrolled in this study.

Additional file 8: Table S2. Primer sequence in this study

\section{Abbreviations}

HOXB9: Homeobox B9; HOX: Homeobox; IL6: Interleukin-6; VEGF: Vascular endothelial growth factor; MTT: 2,3-bis (2-methoxy-4-nitro-5-sulfophenyl)-2Htetrazolium-5-carboxanilide inner salt; EdU: 5-ethynyl-2'-deoxyuridine; AREG: Amphiregulin; ERG: Epiregulin; bFGF: basic fibroblast growth factor; IL8: Interleukin-8; ISP: Intrasplenic injection; DFS: Disease-free survival; OS: Overall survival; shRNA: short hairpin RNA; qRT-PCR: quantitative reverse transcription- polymerase chain reaction; TGF: Transforming growth factor.

\section{Competing interests}

Yuko Kitagawa (Keio University) received research support from Chugai Pharmaceutical Co Ltd. The remaining authors disclose no conflicts.

\section{Authors' contributions}

$\mathrm{TH}, \mathrm{MO}, \mathrm{MW}, \mathrm{HJ}$, and $\mathrm{HH}$ designed the experiments. $\mathrm{YH}$ and $\mathrm{HA}$ conducted the majority of the experiments. NC and HS performed mouse experiments. $\mathrm{MO}$ and MW performed protein assays. HT performed EdU assays. TH, MS, and $Y K$ conceived the project and participated in experimental design. $\mathrm{YH}$ wrote the manuscript. $\mathrm{TH}, \mathrm{MO}$, and $\mathrm{MW}$ participated in writing the manuscript. All the authors have discussed the results and commented on the manuscript. 


\section{Authors' information}

Y.H., T.H., H.T., A.H., N.C., and M.O. are assistant professors of Keio University, School of Medicine. M.W. and H.J. are instructors of Keio University, School of Medicine. H.H. is an associated professor of Keio University, School of Medicine. M.S. is an associate professor of Harvard Medical School. M.S. and Y.K. are professors of Keio University, School of Medicine.

\section{Acknowledgments}

We thank H. Okazaki for the kind technical assistance with all aspects of this study; K. Miyao for the technical help with the protein assays; Dr. S. Sagae for animal management; Dr. T. Endo, Dr. Y. Ishii, Dr. Z. Aisulu, and Dr. K. Shigeta for the helpful discussion; and T. Fukushima for her dedicated involvement in laboratory management. We are grateful to Dr. K. Fukuda for the help with flow cytometry.

This work was supported by a JSPS KAKENHI Grant-in-Aid for Young Scientists (B) (number 22791300) and a Grant-in-Aid for Scientific Research (B) (25293292)

\section{Author details}

${ }^{1}$ Department of Surgery, School of Medicine, Keio University, 35 Shinanomachi, Shinjuku-ku, Tokyo 160-8582, Japan. ${ }^{2}$ Department of Biochemistry, School of Medicine, Keio University, 35 Shinanomachi, Shinjuku-ku, Tokyo 160-8582, Japan. ${ }^{3}$ Department of Laboratory Medicine, School of Medicine, Keio University, 35 Shinanomachi, Shinjuku-ku, Tokyo 160-8582, Japan. ${ }^{4}$ Massachusetts General Hospital Cancer Center, Harvard Medical School, Bldg 149, 13th Street, Charlestown, MA 02129, USA.

Received: 23 October 2013 Accepted: 1 May 2014

Published: 5 May 2014

\section{References}

1. Greer JM, Puetz J, Thomas KR, Capecchi MR: Maintenance of functional equivalence during paralogous Hox gene evolution. Nature 2000, 403:661-665.

2. Taylor HS: The role of HOX genes in the development and function of the female reproductive tract. Semin Reprod Med 2000, 18:81-89.

3. Friedmann $Y$, Daniel CA, Strickland P, Daniel CW: Hox genes in normal and neoplastic mouse mammary gland. Cancer Res 1994, 54:5981-5985.

4. Magli MC, Largman C, Lawrence HJ: Effects of HOX homeobox genes in blood cell differentiation. J Cell Physiol 1997, 173:168-177.

5. Hayashida T, Takahashi F, Chiba N, Brachtel E, Takahashi M, Godin-Heymann N, Gross KW, Vivanco MM, Wijendran V, Shioda T, Sgroi D, Donahoe PK, Maheswaran S: HOXB9, a gene overexpressed in breast cancer, promotes tumorigenicity and lung metastasis. Proc Natl Acad Sci U S A 2010, 107:1100-1105.

6. Chiba N, Comaills V, Shiotani B, Takahashi F, Shimada T, Tajima K, Winokur D, Hayashida T, Willers H, Brachtel E, Vivanco MD, Haber DA, Zou L, Maheswaran S: Homeobox B9 induces epithelial-tomesenchymal transition-associated radioresistance by accelerating DNA damage responses. Proc Natl Acad Sci U S A 2011 109:2760-2765.

7. Bergers $\mathrm{G}$, Benjamin LE: Tumorigenesis and the angiogenic switch. Nat Rev Cancer 2003, 3:401-410.

8. Hanahan D, Folkman J: Patterns and emerging mechanisms of the angiogenic switch during tumorigenesis. Cell 1996, 86:353-364.

9. Folkman J: Role of angiogenesis in tumor growth and metastasis. Semin Oncol 2002, 29:15-18.

10. Willett CG, Boucher Y, Di Tomaso E, Duda DG, Munn LL, Tong RT, Chung DC, Sahani DV, Kalva SP, Kozin SV, Mino M, Cohen KS, Scadden DT, Hartford AC, Fischman AJ, Clark JW, Ryan DP, Zhu AX, Blaszkowsky LS, Chen $H X$, Shellito PC, Lauwers GY, Jain RK: Direct evidence that the VEGFspecific antibody bevacizumab has antivascular effects in human rectal cancer. Nat Med 2004, 10:145-147.

11. O'Connor JP, Carano RA, Clamp AR, Ross J, Ho CC, Jackson A, Parker GJ, Rose CJ, Peale FV, Friesenhahn M, Mitchell CL, Watson Y, Roberts C, Hope L, Cheung S, Reslan HB, Go MA, Pacheco GJ, Wu X, Cao TC, Ross S, Buonaccorsi GA, Davies K, Hasan J, Thornton P, del Puerto O, Ferrara N, van Bruggen N, Jayson GC: Quantifying antivascular effects of monoclonal antibodies to vascular endothelial growth factor: insights from imaging. Clin Cancer Res 2009, 15:6674-6682.
12. Warren RS, Yuan H, Matli MR, Gillett NA, Ferrara N: Regulation by vascular endothelial growth factor of human colon cancer tumorigenesis in a mouse model of experimental liver metastasis. J Clin Invest 1995, 95:1789-1797.

13. Bagri A, Berry L, Gunter $B$, Singh $M$, Kasman I, Damico LA, Xiang $H$, Schmidt M, Fuh G, Hollister B, Rosen O, Plowman GD: Effects of anti-VEGF treatment duration on tumor growth, tumor regrowth, and treatment efficacy. Clin Cancer Res 2010, 16:3887-3900.

14. Seki H, Hayashida $T$, Jinno $H$, Hirose $S$, Sakata M, Takahashi M, Maheswaran S, Mukai M, Kitagawa Y: HOXB9 expression promoting tumor cell proliferation and angiogenesis is associated with clinical outcomes in breast cancer patients. Ann Surg Oncol 2012, 19:1831-1840

15. Gossen M, Bujard H: Tight control of gene expression in mammalian cells by tetracycline-responsive promoters. Proc Natl Acad Sci USA 1992 89:5547-5551

16. Abate-Shen C: Deregulated homeobox gene expression in cancer: cause or consequence? Nat Rev Cancer 2002, 2:777-785.

17. Jain RK: Molecular regulation of vessel maturation. Nat Med 2003, 9:685-693.

18. Shrestha B, Ansari Kl, Bhan A, Kasiri S, Hussain I, Mandal SS: Homeodomaincontaining protein HOXB9 regulates expression of growth and angiogenic factors, facilitates tumor growth in vitro and is overexpressed in breast cancer tissue. FEBS J 2012, 279:3715-3726.

19. Venook AP, Niedzwiecki D, Lopatin M, Ye X, Lee M, Friedman PN, Frankel W, Clark-Langone K, Millward C, Shak S, Goldberg RM, Mahmoud NN, Warren RS, Schilsky RL, Bertagnolli MM: Biologic determinants of tumor recurrence in stage II colon cancer: validation study of the 12-gene recurrence score in cancer and leukemia group B (CALGB) 9581. J Clin Oncol 2013, 31:1775-1781

20. Chang HY, Nuyten DS, Sneddon JB, Hastie T, Tibshirani R, Sorlie T, Dai H, He YD, van't Veer LJ, Bartelink H, van de Rijn M, Brown PO, van de Vijver MJ: Robustness, scalability, and integration of a wound-response gene expression signature in predicting breast cancer survival. Proc Natl Acad Sci USA 2005, 102:3738-3743.

21. Kim H, Watkinson J, Varadan V, Anastassiou D: Multi-cancer computational analysis reveals invasion-associated variant of desmoplastic reaction involving INHBA, THBS2 and COL11A1. BMC Med Genomics 2005, 3:51.

22. Novick D, Shulman LM, Chen L, Revel M: Enhancement of interleukin 6 cytostatic effect on human breast carcinoma cells by soluble IL- 6 receptor from urine and reversion by monoclonal antibody. Cytokine 1992, 4:6-11

23. Chung $Y C$, Chaen $Y L$, Hsu CP: Clinical significance of tissue expression of interleukin- 6 in colorectal carcinoma. Anticancer Res 2006, 26:3905-3911.

24. Plante M, Rubin SC, Wong GY, Federici MG, Finstad CL, Gastl GA: Interleukin-6 level in serum and ascites as a prognostic factor in patients with epithelial ovarian cancer. Cancer 1994, 73:1882-1888.

25. Poutahidis T, Haigis KM, Rao VP, Nambiar PR, Taylor CL, Ge Z, Watanabe K, Davidson A, Horwitz BH, Fox JG, Erdman SE: Rapid reversal of interleukin-6dependent epithelial invasion in a mouse model of microbially induced colon carcinoma. Carcinogenesis 2007, 28:2614-2623.

26. Yu H, Pardoll D, Jove R: STATs in cancer inflammation and immunity: a leading role for STAT3. Nat Rev Cancer 2009, 9:798-809.

27. Bates DO, Catalano PJ, Symonds KE, Varey AH, Ramani P, O'Dwyer PJ, Giantonio BJ, Meropol NJ, Benson AB, Harper SJ: Association between VEGF splice isoforms and progression-free survival in metastatic colorectal cancer patients treated with bevacizumab. Clin Cancer Res 2012, 18:6384-6391.

28. Maru D, Venook AP, Ellis LM: Predictive biomarkers for bevacizumab: are we there yet? Clin Cancer Res 2013, 19:2824-2827.

29. Ha TU, Segev DL, Barbie D, Masiakos PT, Tran TT, Dombkowski D, Glander M, Clarke TR, Lorenzo HK, Donahoe PK, Maheswaran S: Mullerian inhibiting substance inhibits ovarian cell growth through an $\mathrm{Rb}$-independent mechanism. J Biol Chem 2000, 275:37101-37109.

30. Leconte A, Garambois V, Ychou M, Robert B, Pourquier D, Terskikh A Mach JP, Pelegrin A: Involvement of circulating CEA in liver metastases from colorectal cancers re-examined in a new experimental model. Br J Cancer 1999, 80:1373-1379. 
31. Handa K, Ohmura M, Nishime C, Hishiki T, Nagahata Y, Kawai K, Suemizu H, Nakamura M, Wakui M, Kitagawa Y, Suematsu M, Tsukada K: Phosphorescence-assisted microvascular $\mathrm{O}_{2}$ measurements reveal alterations of oxygen demand in human metastatic colon cancer in the liver of superimmunodeficient NOG mice. Adv Exp Med Biol 2010, 662:423-429.

doi:10.1186/1476-4598-13-102

Cite this article as: Hoshino et al: Bevacizumab terminates homeobox B9-induced tumor proliferation by silencing microenvironmental communication. Molecular Cancer 2014 13:102.

\section{Submit your next manuscript to BioMed Central and take full advantage of:}

- Convenient online submission

- Thorough peer review

- No space constraints or color figure charges

- Immediate publication on acceptance

- Inclusion in PubMed, CAS, Scopus and Google Scholar

- Research which is freely available for redistribution 